\title{
Phosphor materials under high-density XUV FEL excitation: mechanisms of luminescence quenching
}

\author{
Sebastian Vielhauer ${ }^{*}$, Vladimir Babin ${ }^{\mathrm{a}}$, Marco De Grazia ${ }^{\mathrm{b}}$, Eduard Feldbach ${ }^{\mathrm{a}}$, Marco Kirm ${ }^{\mathrm{a}}$, Vitali \\ Nagirnyi $^{\mathrm{a}}$, Andrei N. Vasil'ev ${ }^{\mathrm{c}}$ \\ ${ }^{a}$ Institute of Physics, University of Tartu, Riia 142, 51014 Tartu, Estonia; \\ ${ }^{\mathrm{b}} \mathrm{CEA} / \mathrm{DSM} / \mathrm{DRECAM} /$ Service des Photons, Atomes et Moleculés, CEA-SACLAY, 91191 Gif-sur- \\ Yvette, France; \\ ${ }^{c}$ Faculty of Physics, Moscow State University, Moscow, 119992, Russia
}

\begin{abstract}
Photoluminescence of scintillator materials based on intrinsic excitonic luminescence $\left(\mathrm{PbWO}_{4}\right)$, and on extrinsic luminescence from doped trivalent rare earth ions $\left(\mathrm{RE}^{3+}\right)$, such as $\mathrm{Y}_{3} \mathrm{Al}_{5} \mathrm{O}_{12}: \mathrm{Ce}^{3+}$ and $\mathrm{Lu}_{3} \mathrm{Al}_{5} \mathrm{O}_{12}: \mathrm{Pr}^{3+}$ was studied under excitation with free electron laser (FEL) light in the 50-100 eV energy range. In case of $\mathrm{PbWO}_{4}$, non-exponential behavior in the initial part of decay curves was observed depending on the FEL pulse energy, and modeled in terms of the bimolecular self-quenching process. For the $\mathrm{RE}^{3+}$ doped samples, a reduction in light yield with increasing pulse energy is observed, which can be traced to saturation of the available $\mathrm{RE}^{3+}$ sites in the crystal due to the initial high concentration of electron-hole pairs after FEL excitation.
\end{abstract}

Keywords: Photoluminescence, scintillators, Free Electron Laser, XUV, self-quenching, tungstates, rare earth ions

\section{INTRODUCTION}

With the development of powerful sources for the XUV to X-ray energy range based on FELs or higher harmonics generation (HHG) lasers, wide band gap materials used as phosphors or scintillators will be exposed to excitation densities much higher than in conventional sources such as synchrotrons [1]. When used under these conditions, e.g. in detectors for beam diagnostics or experimental setups, the response characteristics and long-term stability need to be known to allow for accurate measurements [2][3]. In a first step to study these factors for various phosphors, we investigated time-resolved photoluminescence for various wide band-gap materials with both intrinsic (excitonic) luminescence, and extrinsic emission from trivalent rare earth ions $\left(\mathrm{RE}^{3+}\right)$ doped into the crystal. The samples were excited using femtosecond FEL pulses at the FLASH facility in Hamburg with photon energies of 48 and $89 \mathrm{eV}$.

In this energy range, the incident photons from the excitation pulses are absorbed within a thin layer (absorption coefficients in the range $10^{-5} \mathrm{~cm}^{-1}$ ), where they create electron-hole pairs in the crystals. Due to the relatively high kinetic energy of the electrons, secondary electron-hole pairs are created through inelastic scattering. In case of intrinsic emission, the thermalized electrons and holes form self-trapped excitons (STE), which can than recombine radiatively. In case of $\mathrm{RE}^{3+}$-doped systems, the energy of the electron-hole pairs is transferred to excite the dopant ion, which consequently relaxes from its excited state under emission of photons. In case of intra-configurational $4 \mathrm{f}$ transitions in $\mathrm{RE}^{3+}$ ions, the lifetime of these states is usually in the microsecond range, whereas spin-allowed transitions from $5 \mathrm{~d}$ to $4 \mathrm{f}$ states, e.g. in the commonly used $\mathrm{Ce}^{3+}$, have typical lifetimes in the sub-microsecond range.

When dealing with excitation sources with high pulse energies, two different aspects have to be taken into account that can influence the light yield. In case of the $\mathrm{RE}^{3+}$ doped systems, a saturation of the available RE ions may occur, since the doping concentration of these ions is usually limited to 1 to $5 \mathrm{~mol} \%$ due to concentration quenching [4]. If more electron-hole pairs are created in a volume than there are available dopant ions, trapping on defects and unwanted impurities is increased, which affects the light yield for the $\mathrm{RE}^{3+}$ emission. For crystals with intrinsic STE emission as the dominant luminescence channel, high excitation intensity leads to a high initial concentration of STE states. Unlike low-density excitation, where STEs can be treated as isolated states, the interaction between the excitons under high-

*sebastian.vielhauer@ut.ee; http://www.fi.ut.ee

Damage to VUV, EUV, and X-Ray Optics II, edited by Libor Juha, Saša Bajt, Ryszard Sobierajski Proc. of SPIE Vol. 7361, 73610R - (C) 2009 SPIE · CCC code: 0277-786X/09/\$18 · doi: 10.1117/12.822292 
density excitation reduces the number of radiatively recombining excitons significantly. This process leads to a characteristic shortening of lifetimes in the initial part of luminescence decay curves.

\section{EXPERIMENTAL}

The experiments were carried out at the beamline BL1 of the free electron laser facility FLASH of DESY in Hamburg, Germany. During our experiments, the photon energy was 48.43 or $89.84 \mathrm{eV}$ with pulse durations of $\sim 25 \mathrm{ps}$ and a $5 \mathrm{~Hz}$ repetition rate. The elliptic spot on the samples was calculated to be $150 \times 250 \mu \mathrm{m}( \pm 50 \mu \mathrm{m})$ in size for the geometry of our experimental chamber [5].

For the measurements, the crystalline samples were mounted in a UHV experimental chamber after gluing them on the cold finger of a liquid He cryostat, allowing to measure luminescence both at $8 \mathrm{~K}$ and $300 \mathrm{~K}$. Emission spectra were recorded using an ARC SP308i UV/visible spectrograph in combination with a Princeton Instruments CCD detector. The spectra were recorded over $\sim 2000$ pulses, and the average pulse intensity was adjusted using the gas attenuator. Decay spectra were measured using an XP2020Q photomultiplier tube (PMT) pointing directly towards the sample in combination with suitable glass filters to select specific emission bands.

The decay spectra were recorded in single shot experiments by measuring the photocurrent with a fast LeCroy oscilloscope. For each decay spectrum, a reference signal from a micro-channel plate detector mounted in the beamline was recorded in parallel, determining the pulse energy delivered for each shot. This allowed averaging over decay curves measured under comparable pulse energies to reduce the noise level of the single shot measurements. The reference pulse heights were correlated to beam energies with the help of the beam energies recorded in the FLASH database.

In case of optimal FEL performance, we could reach maximum pulse energies on the sample up to $25 \mu \mathrm{J}$ at $89.84 \mathrm{eV}$, which corresponds to $1.7 \cdot 10^{12}$ photons at this photon energy. For the luminescence spectra, which were recorded over 2000 FEL pulses, the $\mathrm{N}_{2}$ gas attenuator built into the FEL beamline allowed the reduction of the pulse energy to less than $10 \%$ of the unattenuated beam. For the decay spectra, where also the pulse-to-pulse fluctuations of the FEL due to the stochastic SASE process were taken into account [1], an energy range between $\sim 5-100 \%$ of the maximal pulse intensity was available.

\section{EXITATION DENSITY EFFECTS ON THE LUMINSCENCE FROM PbWO}

\subsection{Emission and decay spectra for $\mathrm{PbWO}_{4}$ under FEL excitation}

Fig. 1 shows emission spectra for $\mathrm{PbWO}_{4}$ recorded at $8 \mathrm{~K}$ and $300 \mathrm{~K}$ and photon energy of $89.84 \mathrm{eV}$ for different average pulse energies. The observed shift in the emission bands between $8 \mathrm{~K}$ and room temperature are typical for $\mathrm{PbWO}_{4}$ crystals with oxygen deficiency sites. At low temperatures, "blue" excitonic luminescence from the lowest triplet STE state of $\mathrm{WO}_{4}{ }^{2-}$ complex anions dominates the emission [6]. At room temperature however, the observed "green" luminescence band is ascribed to excitons localized at oxygen-deficient $\mathrm{WO}_{3}$ sites [7]. Comparing the three emission spectra measured for different average pulse energies, no significant changes in the structure of the bands were observed. This corresponds to similar findings in $\mathrm{CaWO}_{4}$ and $\mathrm{CdWO}_{4}[2]$ with the exception that in the case of $\mathrm{CaWO}_{4}$, a weak band of impurity-related defect emission at around $600 \mathrm{~nm}$ was observed. This defect band showed signs of saturation under higher pulse energies, which can easily be explained by the comparably low concentration of impurities in the crystal.

Decay curves for the blue/green emission in $\mathrm{PbWO}_{4}$ for $8 \mathrm{~K}$ and $300 \mathrm{~K}$ are shown in Fig. 2 for different excitation pulse energies. The decay spectra recorded under FEL excitation exhibit a significant departure from the single-exponential behavior in the initial part ( $\sim 2 \mu \mathrm{s}$ at $8 \mathrm{~K}$ and $\sim 6 \mu$ s at $300 \mathrm{~K})$. This non-exponential part becomes more pronounced with increasing pulse energy, as can be seen from Fig. 3b, where the ratio of peak intensity (at decay time $t=0$ ) and the integrated area of the decay curves for different pulse energies is shown. In this figure, also the integrated area under the decay curves as a function of pulse energy is plotted; it is clearly seen that the range for proportionality (i.e. a constant

Proc. of SPIE Vol. 736173610 R-2 


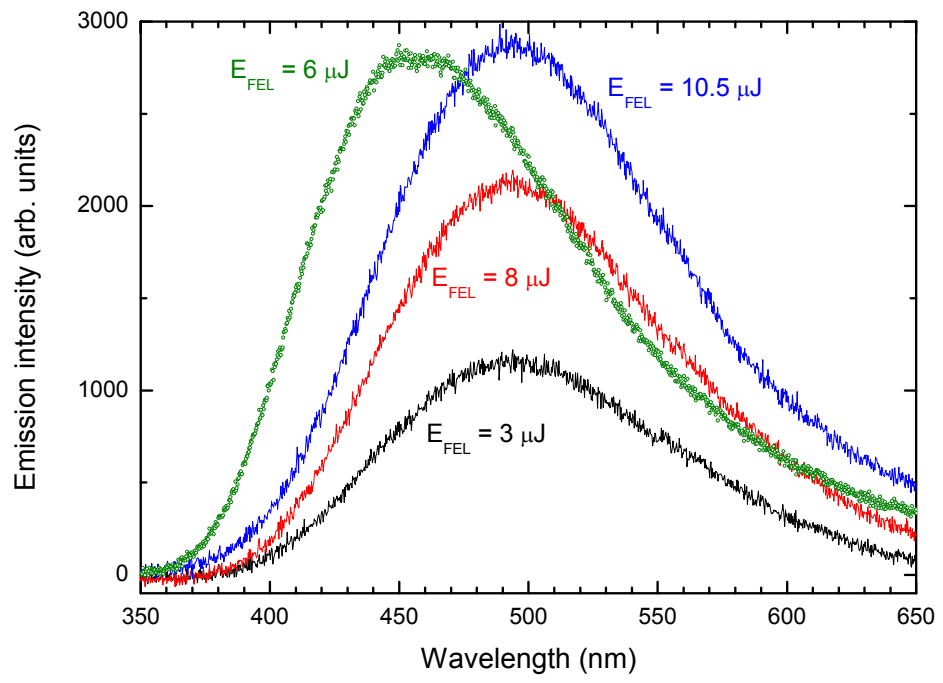

Fig. 1. Emission spectra for $\mathrm{PbWO}_{4}$ measured at $8 \mathrm{~K}$ (dots) and $300 \mathrm{~K}$ (lines) under FEL excitation with photon energy of $89.84 \mathrm{eV}$ using different attenuation levels. The indicated pulse energies are averages over the period of measurements. Note that the intensity of the emission spectrum at $8 \mathrm{~K}$ is not scalable with the room temperature spectra.

light yield) is limited to lower pulse energies of less than $7 \mu \mathrm{J}$. At longer decay times, the decay curves show singleexponential decay behavior with radiative lifetimes in agreement with literature data [7][8]. The observed decay structures under FEL excitation in $\mathrm{PbWO}_{4}$ are similar to $\mathrm{CaWO}_{4}$ and $\mathrm{CdWO}_{4}$ under FEL and $\mathrm{HHG}$ excitation [9][10].

\subsection{Self-quenching of excitons}

The observed deviation from a single exponential decay behavior in the initial part of the decay curves in $\mathrm{CaWO}_{4}$ and $\mathrm{CdWO}_{4}$ under high excitation densities has been explained by the model of excitonic self-quenching [2][11]. The model is described in detail in these references, and will be only briefly introduced here. It is assumed that the incident exciting light spot is of circular shape with radius $a$, and that it follows a Gaussian intensity distribution. The photons are absorbed in the crystal, resulting in an exponential decrease of photon density with an absorption coefficient $\alpha$. Since the photon energy is many times higher than the band-gap energy of the crystal, we have to assume that several secondary electron-hole pairs are created through inelastic scattering. The number of STEs created per incident photon is $\sigma$. After self-trapping of the electron-hole pairs, we can describe an initial density $n_{0}(\rho, z, t=0)$ of the STEs for the radial coordinate $\rho$ and the depth $z$ in the crystal using the expression

$$
n_{0}(\rho, z, 0)=I_{0} \frac{\alpha \sigma}{\pi a^{2}} e^{-\rho^{2} / a^{2}-\alpha z},
$$

where $I_{0}$ is the number of incident photons. Since the actual spot shape during the experiments was of elliptic form, a circular area of the same size was approximated. Based on visible surface damage on some of the samples, the sample spot was reconsidered to be about $350 \times 45 \mu \mathrm{m}$ instead of the $250 \times 150 \mu \mathrm{m}$ that was obtained through calculations for the FEL beam properties and the optics of the beamline. The equivalent radius for a circular spot with the same base area is $62.5 \mu \mathrm{m}$ for the experimentally obtained spot dimensions. 


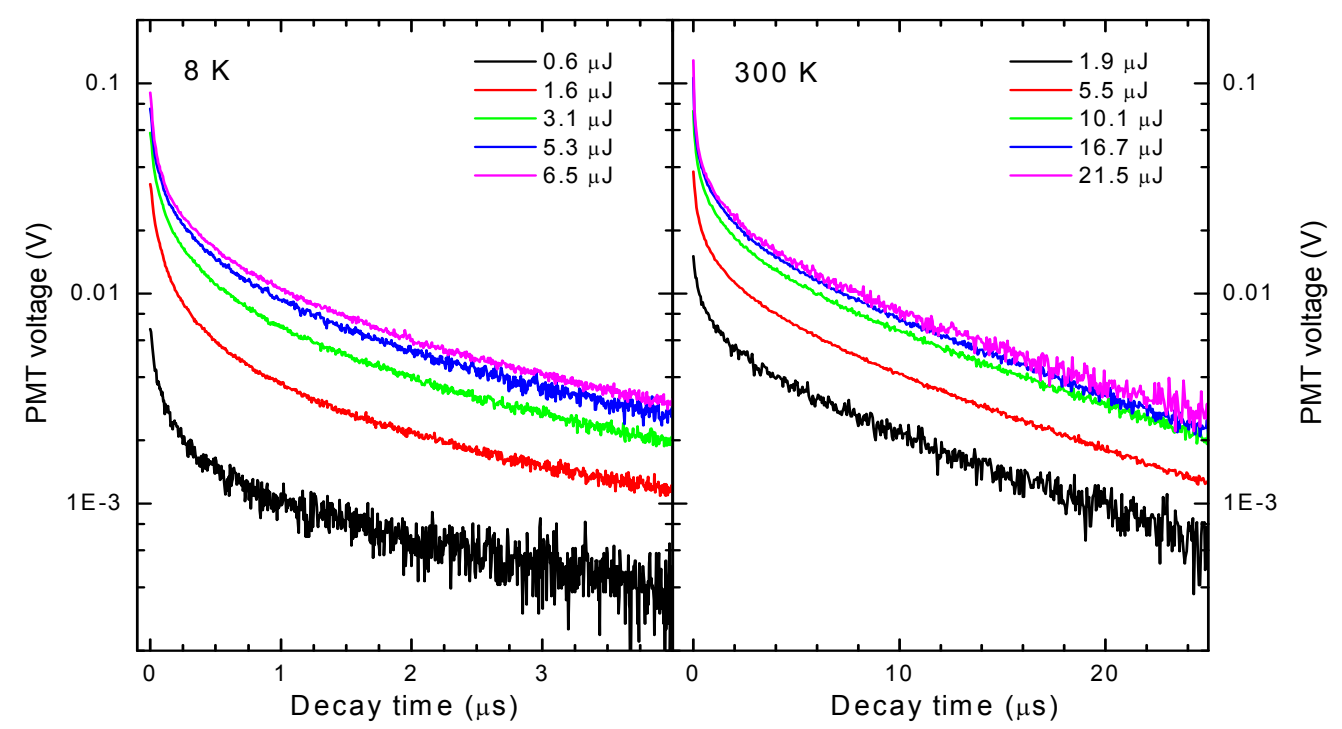

Fig. 2. Decay curves of $\mathrm{PbWO}_{4}$ for different FEL pulse energies at $8 \mathrm{~K}$ (left) and $300 \mathrm{~K}$ (right). Photon energy for the FEL was $89.84 \mathrm{eV}$. Note the different time scales.

Excitons in close proximity are subjected to dipole-dipole interaction processes. These processes, which depend on the local density $n(\rho, z, t)$, lead to energy transfer of one STE (which combines non-radiatively) to its neighbor, resulting in a single, highly excited exciton, which will consequently relax to the ground state, or an unbound electron-hole pair. In the latter case, the charge carriers will quickly be trapped to form a STE again. The net effect of the dipole-dipole interaction can be described in the following way: STE + STE -> STE* $>$ STE in the first case, or STE + STE $->e-h->$ STE in the second. Assuming that the population of STEs is only effected by radiative decay with lifetime $\tau_{r}$ and the selfquenching effect as outlined above, we can formulate a rate equation for the local density of STEs $n(r, t)$ at a given time $t$ of the form

$$
\frac{\partial n(\mathbf{r}, t)}{\partial t}=-\frac{n(\mathbf{r}, t)}{\tau_{r}}-\gamma(t) n^{2}(\mathbf{r}, t),
$$

where the first term on the left hand describes the change of density due to radiate decay, and the second, which is quadratic in $n$, considers the self-quenching of two neighboring STEs. It uses the bimolecular rate of reaction $\gamma(t, r)$, which describes the probability of self-quenching in terms of dipole-dipole interaction radius $R_{d_{-} d}$ as

$$
\gamma(t, \mathbf{r}) \equiv \gamma(t)=\frac{q_{d-d}}{2} \frac{R_{d-d}^{3}}{\sqrt{t \tau_{r}}}, \quad q_{d-d}=\frac{4 \pi^{3 / 2}}{3}=7.42 .
$$

The spatial dependency $\boldsymbol{r}$ of $\gamma(t, \boldsymbol{r})$ needs to be considered only in case of inhomogeneous distribution of STEs over the effective interaction distance, which is on the nanometer scale. In our case with a spot size in of the order of $100 \mu \mathrm{m}$, this correlation dependency can be neglected.

The differential equation defined in equations (2) and (3) can be solved in a closed form when we assume an initial distribution of STE at $t=0$ as defined in equation (1). As a result, we find the luminescence intensity $I_{\text {lum }}$ at a given time $t$ is of the form

$$
I_{\text {lum }}(t)=\frac{\sigma I_{0}}{\tau_{r}} e^{-t / \tau_{r}} \frac{-\mathrm{Li}_{2}\left(-2 \pi^{2} N_{0}^{\max } R_{d-d}^{3} \operatorname{erf}\left(\sqrt{t / \tau_{r}}\right) / 3\right)}{2 \pi^{2} N_{0}^{\max } R_{d-d}^{3} \operatorname{erf}\left(\sqrt{t / \tau_{r}}\right) / 3} .
$$




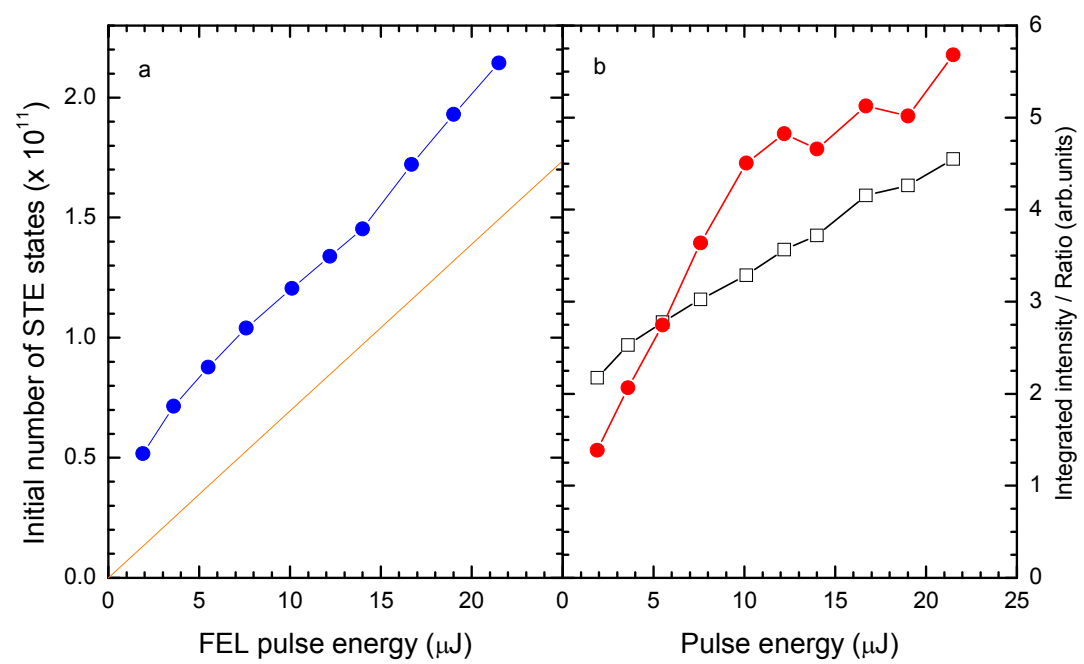

Fig. 3: a: Resulting values for the initial number of STE states as a function of FEL pulse energy from fitting the selfquenching model to the experimental decay curves of $\mathrm{PbWO}_{4}$ luminescence measured at $300 \mathrm{~K}$ (filled circles). The line indicates the numbers of incident photons for the given FEL pulse energy. b: Area under the decay curves (integrated intensity) measured at $300 \mathrm{~K}$ for different pulse energies (filled circles). The open squares indicate the ratio between the peak intensity of the decay curves at $t=0$ and the integrated intensity.

Here we use the dilogarithm function $\operatorname{Li}_{2}(x)$ and the maximal concentration of excitons at $r=z=t=0$ of the form $N_{0}^{\max }=I_{0} \frac{\alpha \sigma}{\pi a^{2}}$.

\subsection{Fit of the model to the measured decay curves}

With the closed formula (4), we can now try to model our measured decay curves. Using the same approach as in [2], two parameters $\left(\tau_{r}\right.$ and $R_{d-d}$ ) were fitted using the full data set of 10 decay curves, and a third parameter, $I_{0} \cdot \sigma$, was optimized for each individual curve. For the absorption coefficient, $\alpha=10^{5} \mathrm{~cm}^{-1}$ was used and a radius of $a=62.5 \mu \mathrm{m}$ as discussed above. The values for $\tau_{r}$ were included into the optimization because the radiative lifetime depends significantly on the sample. The initial intensity $I_{0}$ is always coupled to the STE yield factor $\sigma$, so we consider $I_{0} \cdot \sigma$ as the relevant parameter for the fitting process. Note that for $\sigma$ only an upper limit can be given as we can create up to $\sigma_{\max }$ $=E_{p h} / E_{G}$ electron-hole pairs for a given photon energy $E_{p h}$ and the band gap energy $E_{G}$. In this case we have to make the unrealistic assumption that no energy is lost to the lattice throughout the whole $e-h$ multiplication process.

The fit results are shown in Fig. 4a for the room temperature spectra; good agreement between the model curves and the experimental data is observed. For the two common fit parameters, values of $\tau_{r}=10 \mathrm{~ns}$ and $R_{d-d}=2.2 \mathrm{~nm}$ were calculated. The value of $\tau_{r}$ defines the single-exponential part of the decay curves, so that the agreement with the measured curves can be directly seen in Fig. $4 \mathrm{a}$ at longer decay times. The dipole-dipole interaction radius $R_{d-d}$ of $2.2 \mathrm{~nm}$ is comparable to previously obtained results (based on the same theoretical approach) for $\mathrm{CaWO}_{4}$ of $2.9 \mathrm{~nm}$ [2] and $\mathrm{CdWO}_{4}$ of $4.3 \mathrm{~nm}[11]$. The results for the fit parameter $I_{0} \cdot \sigma$, which were adjusted to each individual decay curve, are shown in Fig. 3a. The straight line in the figure indicates the number of exciting photons $I_{0}$ for the given pulse energy with $89.84 \mathrm{eV}$ photons. The fitted values follow the same linear trend, indicating a value of $\sigma$ close to one, but with a band gap energy of $\sim 4 \mathrm{eV}$ in $\mathrm{PbWO}_{4}$ [12], a much higher value of $\sigma$ can be expected, since $\sigma_{\max }=E_{p h} / E_{G} \approx 22$. This deviation between fitted values for $\sigma$ and the expected ones is probably related to the simple theoretical approach and the given uncertainties for the spot radius and the absorption coefficient.

Since the increasing influence of the self-quenching with initial STE concentrations $N_{0}^{\max }$ is less pronounced over the range of pulse energies available in the experiment, Fig. $4 \mathrm{~b}$ shows extrapolated decay curves calculated for the same fit parameters, but for a wide range of $N_{0}^{\max }$. The lowest curve for $N_{0}^{\max }=3 \times 10^{9} \mathrm{~cm}^{-3}$ shows nearly no deviation from single-exponential decay, whereas for the highest value of $3 \times 10^{15} \mathrm{~cm}^{-3}$, the self-quenching leads to a reduction of inten- 


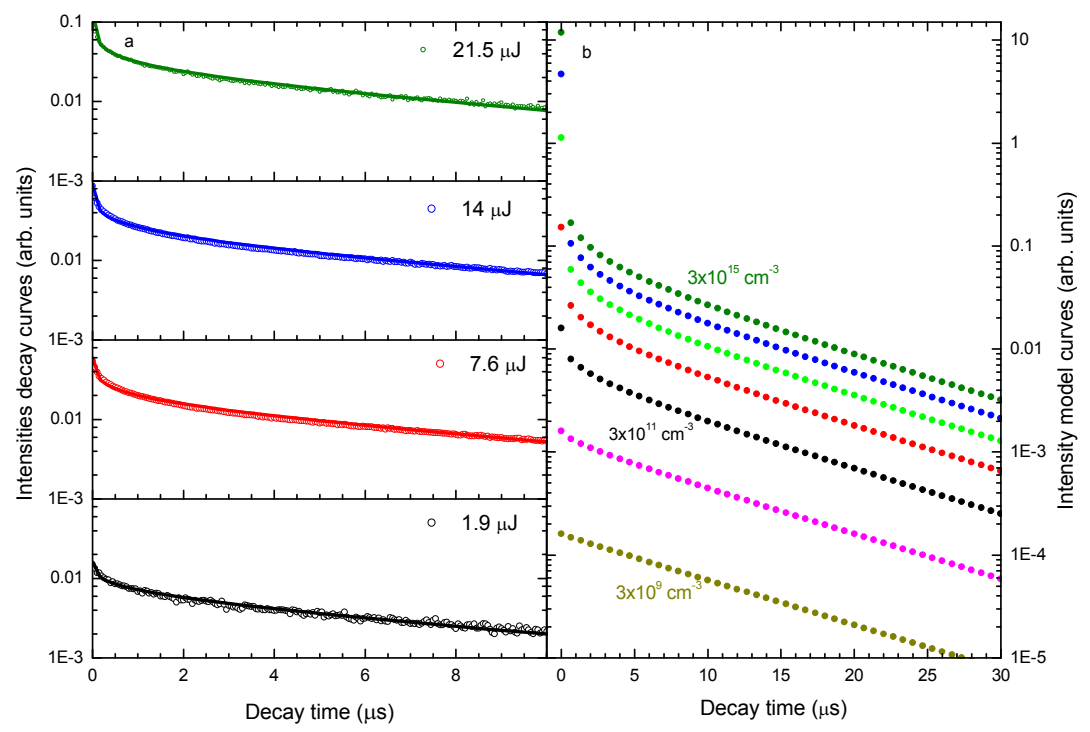

Fig. 4: a: Fit results (lines) for the self-quenching model for decay curves of $\mathrm{PbWO}_{4}$ STE luminescence at $300 \mathrm{~K}$ (circles) measured at different pulse energies as indicated in the figure. b: extrapolation of the model curves for $\mathrm{PbWO}_{4}$ for values of maximum initial concentration $N_{0}{ }^{\max }$ of STE ranging from $3 \times 10^{9} \mathrm{~cm}^{-3}$ (lowest curve) to $3 \times 10^{15} \mathrm{~cm}^{-3}$ (top curve). The limitations due to the damage threshold of the crystal at high densities are not taken into account.

sity by a factor of $\sim 100$ in the first microsecond of the decay. It should be noted though that the highest concentrations, which are $\sim 10^{4}$ times higher than the values fitted to the measured curves, are only possible for pulse energies far above the damage thresholds for this kind of material. Nevertheless, this extrapolation gives a good indication of the sensitivity of the self-quenching process on the initial STE concentration.

\section{RE $^{3+}$ DOPED PHOSPHORS UNDER FEL EXCITATION}

As an example for the luminescence properties of $\mathrm{RE}^{3+}$ doped scintillators, Fig. 5 shows emission spectra and decay curves for $\mathrm{Y}_{3} \mathrm{Al}_{5} \mathrm{O}_{12}$ (YAG) doped with $\mathrm{Ce}^{3+}(5 \mathrm{at} \%)$. The emission spectra were recorded for different transmissions of the gas attenuator, as indicated in the figure. No significant changes are observed in the shape of the spectra, and the general form agrees well with the literature [13][14]. The same findings hold for the decay curves shown for $89.84 \mathrm{eV}$ FEL excitation for two pulse energies at room temperature in the inset of Fig. 5. The stability of both the emission and decay spectra indicate that the luminescence processes remain unchanged under the influence of increasing FEL pulse energy. This stability in term of luminescence properties is characteristic also for the other $\mathrm{RE}^{3+}$ doped samples that were investigated.

A different image appears when we look at the light yield for high excitation densities. In Fig. 6, the peak intensity for decay curves (intensity measured at $t=0$ ) is shown as a function of the FEL pulse energy for YAG:Ce as well as $\mathrm{Lu}_{3} \mathrm{Al}_{5} \mathrm{O}_{12}$ doped with $\mathrm{Ce}^{3+}$ or $\mathrm{Pr}^{3+}$ (LuAG:Ce 2 at\% and LuAG:Pr 5 at\%), and $\mathrm{LiCaAlF}_{6}$ doped with 0.5 at $\% \mathrm{Eu}^{3+}$ (LiCAF:Eu). To better compare the dependencies, the datasets were scaled so that the peak intensities are comparable for an FEL pulse energy of $4 \mu \mathrm{J}$. In all cases, independent of crystal type of $\mathrm{RE}^{3+}$ concentration, the samples show non-linear response with the pulse energy. Using the distribution of electron-hole pairs in the crystal established in equation (1), one can estimate a maximal concentration for $e-h$ pairs $N_{0}^{\max }=0.056 \mathrm{~nm}^{-3}$ for $89.84 \mathrm{eV}$ photons for our geometry and a pulse energy of $1 \mu \mathrm{J}$. In LuAG or YAG, a unit cell contains 24 dodecahedral sites on which a $\mathrm{RE}^{3+}$ ion can be substituted, and the volume of the unit cell corresponds to $1.698 \mathrm{~nm}^{3}$ (LuAG) and $1.728 \mathrm{~nm}^{3}$ (YAG) [15]. 


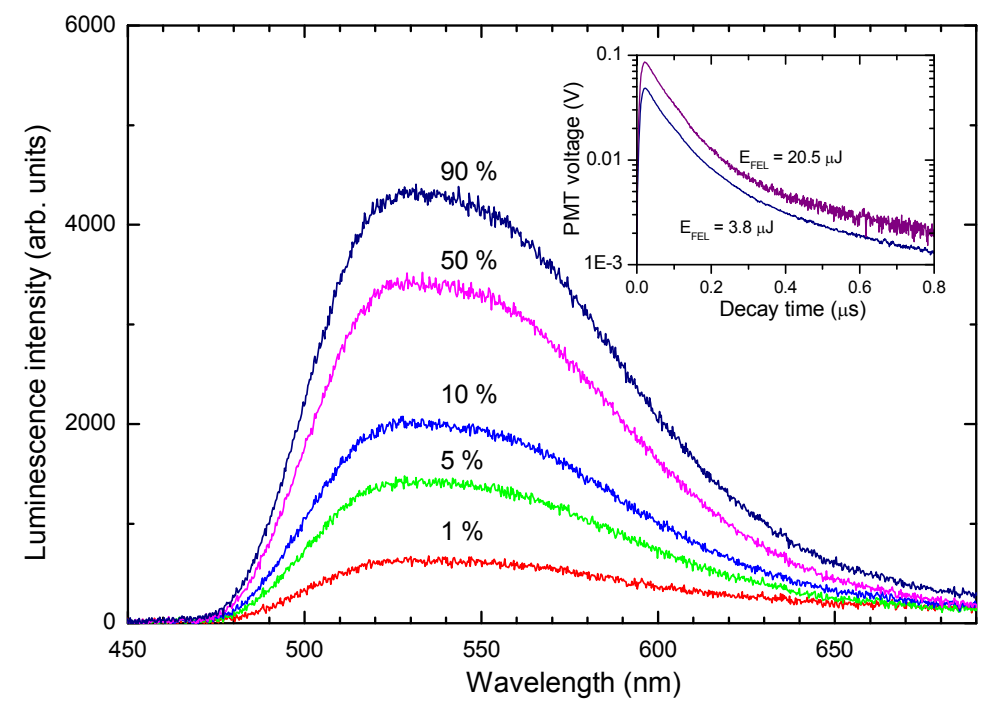

Fig. 5: Emission spectra of YAG:Ce excited with FEL pulses of $48.43 \mathrm{eV}$ photon energy and different transmissions (in per cent) of the gas attenuator, measured at room temperature. The inset shows decay curves for $89.84 \mathrm{eV}$ FEL excitation for two pulse energies, also measured at room temperature.

Based on these estimations, we can expect about one $e-h$ pair per 30 unit cells, or $\sim 730$ substitutional places at the point of maximum excitation density for the YAG crystal. Assuming dopant concentrations between 1 and 5 at $\%$, this leads to a ratio of 7.3 to 36.5 dopant ions per created $e-h$ pair. This calculation of course neglects the effect of multiple electronhole pair creation $(\sigma=1)$. With the band gap energy of $6.5 \mathrm{eV}$ in YAG, a $\sigma$ of $\sim 10$ for $89.84 \mathrm{eV}$ photons is realistic, which would bring the density of $e-h$ pairs to the same order as the dopant concentration ( 0.7 to 3.7 ions per e-h for $1 \mu \mathrm{J}$ pulse energy). Similar calculations can be performed for the other systems. With the concentrations of e-h pairs approaching those of the dopant ions, the point of saturation is reached, leading to the observed reduction in light yield with increasing pulse energy.

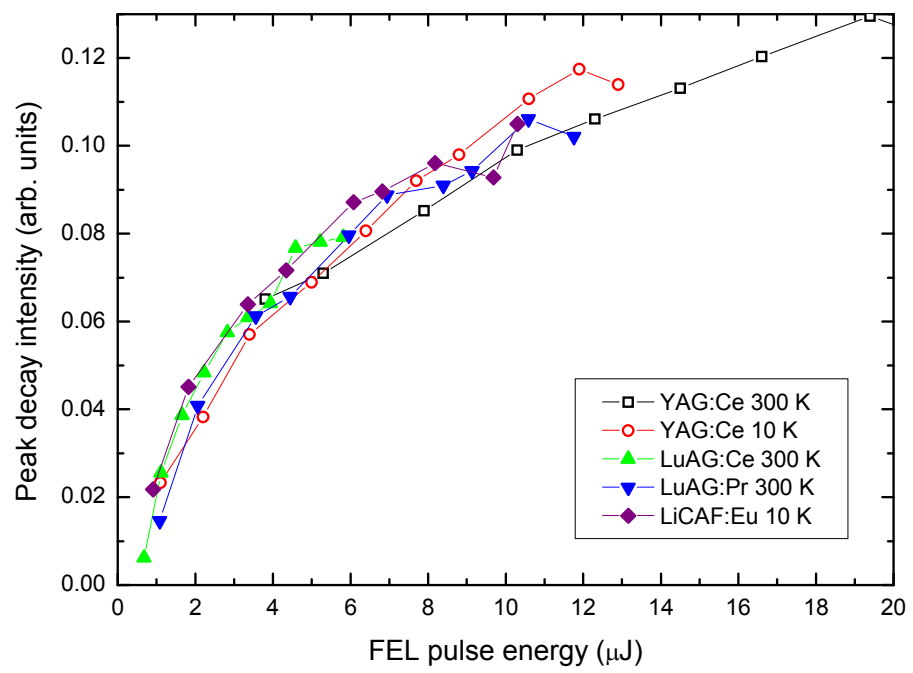

Fig. 6: Dependence of decay intensity (given in terms of the peak intensity in the decay spectra) on the FEL pulse energy for different $\mathrm{RE}^{3+}$ doped materials. The different curves have been normalized to comparable peak intensities for a FEL pulse energy of $4 \mu \mathrm{J}$. All measurements were recorded under excitation with $89.84 \mathrm{eV}$ photons. 


\section{CONCLUSIONS}

Luminescence studies of scintillators under intense XUV FEL excitation have shown that non-linearity due to dipoledipole interaction of closely spaced STEs influence the decay process for $\mathrm{PbWO}_{4}$, but also for other tungstates like $\mathrm{CaWO}_{4}$ and $\mathrm{CdWO}_{4}$. For the pulse energy range available in our experiments, this effect can be reproduced using the self-quenching model as discussed above. In this model, the dipole-dipole interaction radius $R_{d-d}$ describes the critical distance of neighboring STEs for the self-quenching mechanism. In this paper, $R_{d-d}$ was determined to be $2.2 \mathrm{~nm}$ in $\mathrm{PbWO}_{4}$, in a similar way, values for $R_{d-d}$ of $2.6 \mathrm{~nm}$ in $\mathrm{CaWO}_{4}$ and $4.3 \mathrm{~nm}$ in $\mathrm{CdWO}_{4}$ were found in earlier works. While the shape of the decay curves is generally well reproduced, the model tends to underestimate the initial number of created STEs $I_{0} \cdot \sigma$. Several explanations can be given for this discrepancy. First, the model parameters for the spot size and the absorption coefficient, which define the volume for absorption, are only estimates. Since the concentration of STEs is defined both by the number of STEs and the volume of absorption, errors in these parameters feed back to the model results for $I_{0} \cdot \sigma$. Second, the model takes only the most simple of self-quenching processes, the dipole-dipole interaction between two STEs, into account. Especially for very high concentrations, self-quenching processes with more than two STE partners are possible. Finally, the model assumes that no competing processes such as defect emission or non-radiative relaxation occur, which would reduce the number of created STEs.

In the case of the $\mathrm{RE}^{3+}$ doped scintillators, no pulse energy dependent changes of emission or decay spectra were observed in the range of pulse energies available in the experiment. This indicates that the high excitation density does not influence the luminescence process of the $\mathrm{RE}^{3+}$ ion itself. When we look at the luminescence intensity as a function of pulse energy however, it can be clearly seen that the energy transfer from the initially created electron-hole pairs to the dopant ions is strongly reduced for high excitation densities. A comparison between the concentration of dopant ions and initially created $e-h$ pairs shows that already at the lower pulse energies used in our experiments saturation of the $\mathrm{RE}^{3+}$ sites can be achieved.

For both intrinsic excitonic luminescence and dopant luminescence, the weakly focused XUV FEL light that was available in our experiments already affects the performance of the materials. Looking at the future development of FEL sources, such as the Linac Coherent Light Source (LCLS) in Stanford or the European X-Ray Laser Project XFEL in Hamburg, which are currently under development, scintillators may soon be subjected to intense, short-pulsed X-ray radiation. Under these conditions, the high rate of secondary $e-h$ pairs created by the energetic photons will cause similar effects as observed in the XUV range, even for significantly smaller photon densities. For these applications, a good knowledge of the limiting processes in different materials is necessary, and hopefully future studies at higher densities and photon energies will contribute to it.

\section{ACKNOWLEDGEMENTS}

Financial support by the Estonian Science Foundation (grant no. ETF 7274 and 6538), and by the European Community Research Infrastructure Action in the FP6 Program of the EU (Contract RII3-CT-2004-506008 (IA-SFS)) is gratefully acknowledged. Many thanks also to Dr. Rolf Treusch and the team of FLASH for their support during the measurements.

\section{REFERENCES}

[1] Ayvazyan, V. et al., "First operation of a free-electron laser generating GW power radiation at $32 \mathrm{~nm}$ wavelength," Eur. Phys. J. D 37, 297-303 (2006).

[2] Vielhauer, S., Babin, V., De Grazia, M., Feldbach, E., Kirm, M., Nagirnyi, V. and Vasil'ev, A.N., "Self-quenching effects of excitons in $\mathrm{CaWO}_{4}$ under high density XUV FEL excitation," Phys. Sol. State 50, 1789-1794 (2007).

[3] Kirm, M., Andrejczuk, A., Krzywinski, J. and Sobierajski, R. "Influence of excitation density on luminescence decay in $\mathrm{Y}_{3} \mathrm{Al}_{5} \mathrm{O}_{12}$ : $\mathrm{Ce}$ and $\mathrm{BaF}_{2}$ crystals excited by free electron laser radiation in VUV," Phys. Stat. Sol. (c) 2, 649$652(2005)$. 
Yen, W. M., Shionoya, S. and Yamamoto, H., [Phosphor Handbook], 2nd edition, CRC Press Boca Raton, London, New York, 199 (2007).

[5] Treusch, R. (private communication).

[6] Itoh, M. and Sakurai, T., "Composite nature of the self-trapped exciton luminescence in $\mathrm{PbWO}_{4}$," Phys. Stat. Sol. (b) 242, R52-R54 (2005).

[7] Babin, V., Bohacek, P., Krasnikov, A., Nikl, M., Stolovits, A. and Zazubovich, S., "Origin of green luminescence in $\mathrm{PbWO}_{4}$ crystals," J. Lumin. 124, 113-119 (2007).

[8] Laguta, V.V., Nikl, M. and Zazubovich, S., "Luminescence and decay of excitons in lead tungstate crystals," Radiat. Meas. 42, 515-520 (2007).

[9] Kirm, M., Babin, V., Feldbach, E., Nagirnyi, V., Vielhauer, S., Guizard, S., Carré, B., De Grazia, M., Merdji, H., Belsky, A. N., Fedorov, N., Martin, P., "Time resolved luminescence of solids excited by femtosecond VUV pulses and synchrotron radiation," Phys. Stat. Sol. (c) 4, 870-876 (2007).

[10] Belsky, A. N., Carré, B., Fedorov, N., Feldbach, E., Gaudin, J., Guizard, S., Geoffroy, G., De Grazia, M., Kirm, M., Martin, P., Merdji, H., Nagirnyi, V. and Petite, G., "Interaction d'impulsions VUV intenses avec les solides luminescents," J. Phys. IV France 138, 155-161 (2006).

[11] Fedorov, N., Nagirnyi, V., Vasil'ev, A., Belsky, A., Carré, B., Feldbach, E., Gaudin, J., Geoffroy, G., Guizard, S., De Grazia, M., Kirm, M., Martin, P., Merdji, H. and Petite, G., "Utilisation des matériaux luminescents pour la métrologie des faisceaux intenses UVX d'impulsions ultracourtes," J. Phys. IV France 138, 251-257 (2006).

[12] Lacomba-Perales, R., Ruiz-Fuertes, J., Errandonea, D., Martínez-Garzía, D. and Segura, A., "Optical absorption of divalent metal tungstates: Correlation between the band-gap energy and the cation ionic radius", Europhys. Lett. 83, 37002 (2008).

[13] Zych, E., Brecher, C. and Glodo, J., "Kinetics of cerium emission in a YAG:Ce single crystal: the role of traps," J. Phys.: Condens. Matter 12, 1947-1958 (2000).

[14] Mihóková, E., Nikl, M., Mareš, J.A., Beitlerová, A., Vedda, A., Nejezchleb, K., Blažek, K. and D’Ambrosio, C., "Luminescence and scintillation properties of YAG:Ce single crystal and optical ceramics", J. Lumin. 126, 77-80 (2007).

[15] Euler, F., and Bruce, J. A., "Oxygen Coordinates of Compounds with Garnet Structure," Acta Cryst. 19, 971-978 (1965). 